\title{
A CASE OF NON-EUCLIDIAN GEOMETRY.*
}

BY PROF. W. WOOLSEY JOMNSON.

THE note here presented was suggested by the very interesting article by Dr. McClintock in the November number (vol. II., p. 21) of the BuLLETIN, and merely presents in another point of view the pseudo-measurements, occurring in the Cayley interpretation in the case in which $c$ is positive, developed by Dr. McClintock (page $2 \%$ et seq.) under the name of projective distances as distinguished from the real distance of Enclidian geometry.

In the theory of projective metrics, the case is the symmetric one of the opposite variety from that of Lobatschewsky, or that in which the absolute quadric of Cayley is an imaginary sphere.

In the explanation given in the BuLLETIN it will be remembered that, for the geometry of the plane, we have only to assume a central point of the plane, and a sphere of fixed radius touching it at that point; then for any finite straight line or sect in the plane (not simply a sect of a line passing through the central point) the projective measure is the length of the arc of a great circle which is the central projection of the sect upon the spherical surface. In like manner the projective measure of an angle is the spherical angle between the great circles which are the projections upon the sphere of the sides of the given angle. Thus a plane triangle is represented by a certain spherical triangle, and we develop a non-euclidian geometry of two dimensions, of which the theorems are those of spherical geometry.

What the non-euclidian and two-dimensional dwellers in the plane would call the same triangle in different positions would be such triangles as had for projections the same spherical triangle in different positions upon the sphere.

In extending these projective measures to geometry of three dimensions, we seek to establish, with reference to a single assumed central point in space, a consistent system of projective measurement for all lines and angles however situated in space. In doing this we shall establish for every plane in space its central point, and with reference to this point a system of projective measurement in the plane similar to but not identical with that described above. For this purpose Dr. McClintock distinguishes a prime plane in which the projective measurement is defined by means of a tangent sphere whose radius is $r$, and a serondary plane in

\footnotetext{
* Read before the New York Mathemutical Society, March 4, 1893.
} 
which the projective measures are made on a sphere whose radius is still $r$, but whose centre is at a perpendicular distance $p$ from the central point of the plane. Thus in the prime plane the projective distance is equal to the real distance just at the central point, but for the secondary plane the measures are all less in the ratio $r / p$ (less, for in all cases $p>r$ ) than they would be if the sphere retaining the same centre touched the plane.

Having obtained these systems of measurement, Dr. McClintock now dismisses the sphere, and, taking all planes which pass through the "centre of space" as prime planes, shows that every other plane is a secondary plane, its central point being of course the foot of the perpendicular upon the plane from the centre of space. In establishing this it is to be noticed that the projective measure of every sect upon every straight line has been already fixed by the fact that the straight line is in a prime plane, that is, a plane passing through the centre. What we wish to show is that all the projective measurements thus established for lines which lie in a new plane not passing through the centre are consistent with one another in forming a system peculiar to the plane, namely, that above described as belonging to a secondary plane, in which, as stated in the Bulletin, the constant factor (denoted above by $r / p$ ) is a simple function of the perpendicular from the centre of space upon the plane. So that "all planes in space are either prime or secondary, and all projective distances and angles in space are parts of one harmonious system of general geometry of three dimensions."

In studying this elegant result, and obtaining the constant factor referred to above, it occurred to me that additional clearness, from a purely geometrical point of view, would result from the following simple modification of the definitions of projective distances:

Assuming a "central point" $A$ and a linear magnitude $r$, the "projective measure" of a given finite line or "sect" is its apparent magnitude as seen from a point at a distance $r$ from $A$ measured in a direction perpendicular to the plane through the given line and $A$. There is thus a common view-point, as we may call it, or rather a common pair of view-points for all lines lying in a given plane passing through $A$. We may, of course, instead of the apparent magnitude, which is an angle, take the subtending are in the circle whose radius is $r$, but we shall still call this the apparent magnitude. The radius will be always the same, and we are concerned only with the position of the view-point.

Consider now an indefinite straight line. Having found a view-point $P$ as above, it is a view-point for any segment or sect of the line. Pass a plane through $A$ perpendicular to 
the line, and therefore also through the two view-points. Call the point $O$, where this plane cuts the given line, its centre. Then it is obvious that any point of the circle drawn through

Fig. 1.



the view-points from this centre will serve equally well as a view-point. 'That is, from every point of this circle any sect of the line has the same apparent magnitude, which apparent magnitude is its projective measure. We shall call this circle the view-locus of the given line. The view-locus is then a circle of which the given line is the axis; its plane passes through $A$; and, denoting the distance of the line from $A$ by $p$, its radius is $V\left(p^{2}+r^{2}\right)$. Now pass any plane through the given line, and let $A A^{\prime}=d$ be the perpendicular upon it from $A$. Call $A^{\prime}$ the centre of this new plane. 'The extremities $P^{\prime}, P^{\prime}$ of the chord $A A^{\prime}$ produced form a pair of view-points for the given line $O X$, which, with reference to the plane $O A^{\prime} X$, might have been constructed thus: From the centre $A^{\prime}$ of the plane erect a perpendicular $A^{\prime} P^{\prime}=r^{\prime}$, where $r^{\prime 2}=r^{2}+d^{3}$. [since, if $O A^{\prime}=p^{\prime}, r^{\prime 2}+p^{\prime 2}=r^{2}+p^{2}=r^{2}+p^{\prime 2}+d^{2}$ ]. Now, since the position of $A^{\prime}$ and the value of $r^{\prime}$ depend only upon the plane, and not upon the given line $O X$, we have thus found a pair of view-points which will answer for any line in the new plane as well as for $O X$. This new plane is Dr. McClintock's secondary plane; its view-points are further from the plane than those of the prime planes, and are not symmetrically situated with respect to the centre of space.

This establishes the harmonious and consistent system required. There is for each line a view-locus, and for each

Fig. 2.

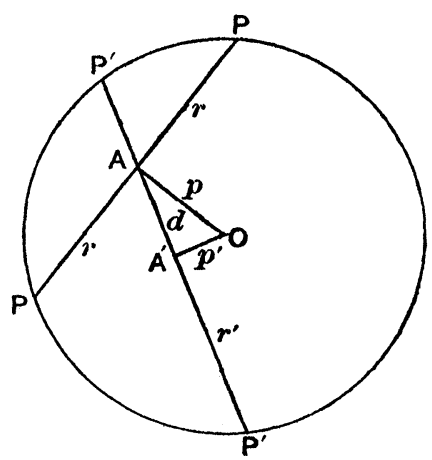
plane a definite view-point. The view-point of a plane is on the view-locus of a line in the plane, so that when a line is regarded as belonging to different planes, the system of apparent or projective measurements assigned to it in the different planes is consistent.

The projective measure of an angle may now be defined, once for all, as its apparent magnitude as seen from the view- 
point of its plane. The definition is unique, and no similar theorem for consistency exists.

Having constructed the view-points of a given plane, by laying off from $A^{\prime}$ (the foot of the perpendicular $A A^{\prime}=d$ ) $A^{\prime} P^{\prime}=r^{\prime}$, where $r^{\prime 2}=r^{2}+d^{2}$, upon the perpendicular; let $C$ be any point of the plane, distant $A C=a$ from the centre of space: then $A^{\prime} C=a^{\prime}$, where $a^{\prime 2}=a^{2}-d^{2}$. Therefore $C P^{\prime 2}=a^{\prime 2}+r^{\prime 2}=a^{2}+r^{2}$. Since this depends only upon the position of $C$, it follows that the view-point of every plane passing through $C$ is at a constant distance from $C$, that is, it lies upon a sphere which we may call the view-sphere of $C$.

The view-locus of a line is a circle having the line as axis, and with reference to which the power of $A$ is $-r^{2}$. The view-sphere of a point is a sphere of which it is the centre, and with reference to which the power of $A$ is $-r^{2}$, while the view-locus of any diameter is the small circle of this sphere whose plane is perpendicular to it and passes through $A$.

When two lines intersect, their view-loci are small circles of the view-sphere of the point of intersection, and these circles intersect in the two view-points of the plane of the intersecting lines.

Again, the view-spheres of two points intersect in a circle, viz., the view-locus of the line joining the points. Those of three points not in a straight line intersect in the two viewpoints of the plane of the three points, and other similar relations might be stated. 